\title{
Estequiometría y ley de conservación de la masa: una relación a analizar desde la perspectiva de los lenguajes químicos
}

\author{
Stoichiometry and the law of mass conservation: \\ aspects to be analyzed from chemical languages
}

Lydia Galagovsky $^{1}$ • Jimena Giudice ${ }^{1}$

\begin{abstract}
Resumen: El presente trabajo se centra en el análisis de la formulación de problemas de estequiometría en diferentes lenguajes químicos que involucran también dibujos con partículas para describir los estados inicial y final de sistemas reaccionantes. El análisis permite tomar conciencia sobre las peculiares restricciones de expresión de algunos de estos lenguajes químicos, así como vislumbrar un problema epistemológico en relación a la aplicación de la ley de conservación de la masa cuando se analizan formatos sintácticos referidos al tema de estequiometría. Concluimos que la enseñanza de los conceptos abstractos y explicaciones químicas fundamentadas en modelos requieren por parte de los docentes una revisión epistemológica sobre sus lenguajes. Esto permitió reflexionar sobre qué decimos y qué queremos significar cuando "hablamos química" y cuando evaluamos a nuestros estudiantes.
\end{abstract}

Palabras clave: Enseñanza de la química. Lenguaje químico. Estequiometría. Conceptos.

\begin{abstract}
The present article is focused on stoichiometry problems written in different chemical languages in which drawings represent atoms behavior at the initial and final state of a reaction. The analysis leads to the need to be aware of the special constraints of each chemical language. A discussion on what teachers say, how they use chemical languages and model-based scientific explanations and how they assess students is presented. These reflections give insights into the meaning of "talking chemistry".
\end{abstract}

Keywords: Chemistry teaching. Chemical language. Stoichiometry. Concepts.

\footnotetext{
${ }^{1}$ Centro de Formación e Investigación en Enseñanza de las Ciencias (CEFIEC), Grupo de Investigación en Aprendizaje y Didáctica de las Ciencias Naturales (GIADiCIeN), Facultad de Ciencias Exactas y Naturales. Universidad de Buenos Aires (UBA), Ciudad Universitaria, Pabellón II, 1428, Buenos Aires, Argentina. E-mail: <lyrgala@qo.fcen.uba.ar>
} 


\section{Introducción}

En trabajos previos hemos discutido cómo elementos presentes en el discurso erudito de química pueden ser origen de obstáculos para el aprendizaje (ALÍ et al., 2010; BEKERMAN, 2006; GALAGOVSKY; BEKERMAN, 2009; GALAGOVSKY; BEKERMAN; DI GIACOMO, 2014; GALAGOVSKY; DI GIACOMO; CASTELO, 2009). En estos trabajos hemos considerado a la clase de química como un espacio de comunicación entre el docente experto (emisor de un mensaje) y los estudiantes novatos (los receptores), con un mensaje a ser comprendido, que es el discurso erudito. La particularidad de esta situación comunicativa en el aula es que el receptor del mensaje, además de decodificarlo apropiadamente -aprehenderlo- debería mostrar que ha aprendido. El emisor deberá entonces evaluar los conocimientos adquiridos por el receptor, referidos al contenido y a la significación del mensaje. Es decir, desde el paradigma de la comunicación (SHANNON; WEAVER, 1949), decodificar un mensaje es interpretarlo correctamente. En el caso de aplicar estos conceptos a la enseñanza de las ciencias, decodificar el mensaje significa, además, que el receptor es capaz de expresar su aprendizaje desde un correcto "hablar ciencia" (LEMKE, 1997). Esto implica un manejo apropiado de los lenguajes, una adecuada interpretación de los modelos científicos, una aplicación correcta a la resolución de ejercicios y una transferencia apropiada sobre problemas nuevos.

El presente trabajo se centra en la formulación de problemas de estequiometría en diferentes lenguajes químicos que involucran dibujos con partículas para describir los estados inicial y final de sistemas reaccionantes. El análisis que desarrollaremos nos permitirá brindar evidencias sobre las peculiares restricciones de expresión de algunos de estos lenguajes químicos, así como vislumbrar un problema epistemológico en relación a la aplicación de la ley de conservación de la masa cuando se analizan formatos sintácticos referidos al tema de estequiometría.

\section{Las respuestas de los alumnos dan el alerta}

Nurrenbern y Pickering (1987) se preguntaron si sus estudiantes de Química General de la Universidades de Kansas City y Wisconsin (EEUU) realmente aprendían una química conceptual, o si sólo aprendían a resolver problemas de tipo algorítmico. Para este análisis utilizaron los resultados que obtuvieron al evaluarlos con dos formatos de preguntas diferentes. En las Figuras 1 y 2 se muestran los ejemplos que estas autoras idearon para evaluar simultáneamente el tema de estequiometría. Denominaremos "problema tradicional" al problema de resolución algorítmica, ya que se trata de un prototipo de pregunta muy utilizada tanto en la enseñanza secundaria (media) como universitaria. Las propias autoras llamaron "conceptual" al tipo de enunciado que requería interpretar dibujos representativos del nivel atómico-molecular. Ellas señalaron que sus estudiantes habían sido enseñados con problemas tradicionales de resolución mediante la aplicación de algoritmos, pero no con los de tipo "conceptual". 
Figura 1. Problemas "tradicional" y "conceptual" utilizados simultáneamente para evaluar a estudiantes en el tema estequiometría

Problema tradicional: Para una mezcla de 2 moles de $\mathrm{H} 2$ y 2 moles de $\mathrm{O}_{2}$ que reaccionan de acuerdo a la siguiente ecuación, ¿cuál es el reactivo limitante y cuántos moles de exceso de reactivo quedarán luego de completada la reacción?

$2 \mathrm{H}_{2}+\mathrm{O}_{2} \rightarrow 2 \mathrm{H}_{2} \mathrm{O}$

Reactivo limitante

A)

B) $\mathrm{O}_{2}$

C)* $\quad \mathrm{H}_{2}$

D) $\quad \mathrm{H}_{2}$

E) No ocurre la reacción porque la ecuación no está balanceada con 2 moles de $\mathrm{H}_{2}$ y 2 moles de $\mathrm{O}_{2}$

Problema conceptual: La reacción del elemento X $(\square)$ con el elemento Y se representa en el siguiente diagrama. ¿ Cuál de las ecuaciones describe mejor esta reacción?

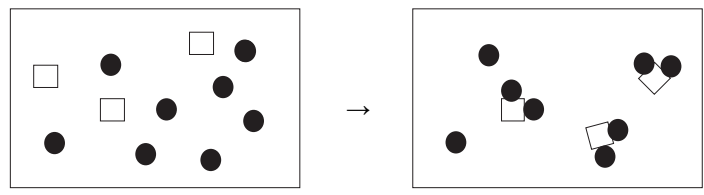

A) $3 \mathrm{X}+8 \mathrm{Y} \rightarrow \mathrm{X}_{3} \mathrm{Y}_{8}$

B) $3 \mathrm{X}+6 \mathrm{Y} \rightarrow \mathrm{X}_{3} \mathrm{Y}_{6}$

C) $* \mathrm{X}+2 \mathrm{Y} \rightarrow \mathrm{XY}_{2}$

D) $3 \mathrm{X}+8 \mathrm{Y} \rightarrow 3 \mathrm{XY}_{2}+2 \mathrm{Y}$

E) $\mathrm{X}+4 \mathrm{Y} \rightarrow \mathrm{XY}_{2}$

Los asteriscos señalan las respuestas correctas.

Fuentes: Nurrenbern; Pickering (1987); Sanger (2005); Sawrey (1990).

Las autoras encontraron que menos del $50 \%$ de los estudiantes que respondieron correctamente el problema "tradicional" contestó satisfactoriamente la pregunta de tipo "conceptual”. Por lo tanto, los estudiantes del primer nivel universitario estarían aprendiendo a resolver problemas de estequiometría mediante cálculos y procedimientos algorítmicos para los cuales se los entrena durante las clases. Sin embargo, no estarían imaginándose qué significan las reacciones a nivel atómico-molecular. Según estas autoras los estudiantes que no pudieron hacer una correspondencia entre los dibujos simbólicos y la ecuación química correspondiente no habrían desarrollado un "pensamiento conceptual" sobre estequiometría. Desde entonces muy frecuentemente se ha mencionado una dicotomía entre el aprendizaje algorítmico y el aprendizaje conceptual en química (TALANQUER, 2012).

Sawrey (1990) repitió esa experiencia con los problemas de la Figura 1 sobre estudiantes del primer año de Química de la Universidad de California, en San Diego (más del 90\% de ellos había cursado uno o más años de Química en su High School. Dicho trabajo mostró que un 66,5\% respondió correctamente el problema "tradicional"; sin embargo, la autora señaló que 
sólo el 11\% de ellos respondió correctamente el problema "conceptual" marcando la opción “C”. El 87\% había elegido la opción incorrecta D.

Nakhleh y Mitchell (1993) reiteraron la experiencia utilizando esta vez los problemas de la Figura 2, sobre estudiantes del primer año de química en Purdue University, Indiana (EEUU).

Figura 2. Problemas "tradicional" y "conceptual" utilizados simultáneamente para evaluar a estudiantes en el tema estequiometría

Problema tradicional: Calcule el máximo peso de $\mathrm{SO}_{3}$ que podría formarse a partir

de 1.9 moles de oxígeno y exceso de azufre, según la ecuación: $2 \mathrm{~S}+3 \mathrm{O}_{2} \rightarrow 2 \mathrm{SO}_{3}$

Problema conceptual: La ecuación siguiente se aplica a la reacción entre azufre y oxígeno: $2 \mathrm{~S}+3 \mathrm{O} 2 \rightarrow 2 \mathrm{SO}_{3}$. Considere que la mezcla de $\mathrm{S}$ y $\mathrm{O}_{2}$ en un contenedor cerrado es la que se ilustra en el siguiente recuadro:
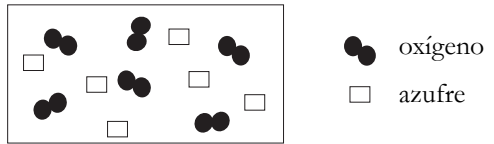

¿Cuál de los siguientes dibujos representa la mezcla final?
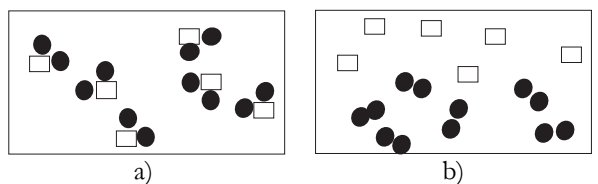

b)

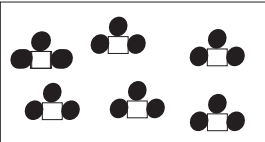

c)

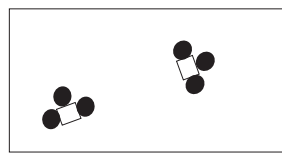

d)

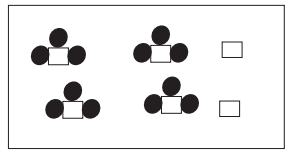

e)

Fuentes: Nakhleh; Mitchell (1993); Nurrenbern; Pickering (1987); Zoller et al. (1995).

Nuevamente, este trabajo reportó que un 50\% de estudiantes mostraron bajas habilidades de tipo "conceptual”, a pesar de pertenecer a la carrera de majors en química y ser buenos estudiantes.

Un estudio comparativo con estudiantes de Purdue y de tres universidades de Israel utilizando el mismo problema de la Figura 2 arrojó resultados similares (ZOLLER et al., 1995).

\section{"Los estudiantes no aprenden conceptualmente"}

Johnstone $(1991,1993,2000)$ ha propuesto que la química involucra tres niveles: el macroscópico, el simbólico y el sub-microscópico. Así, con un nivel en cada vértice, se representa 
el conocido Triángulo de Johnstone. Si bien esta visión ha sido cuestionada (GALAGOVSKY et al., 2003; LABARCA, 2009), sigue siendo un referente hasta nuestros días (TALANQUER, 2012). El nivel sub-microscópico de Johnstone $(1991,1993,2000)$ es aquél donde se dibuja todo lo que podría "verse", si se pudieran percibir los fenómenos químicos a nivel de átomos y moléculas. Por lo tanto, los dibujos de los problemas conceptuales de las figuras 1 y 2 pertenecen al nivel sub-microscópico de Johnstone.

En las investigaciones comentadas previamente (NAKHLEH; MITCHELL, 1993; NURRENBERN; PICKERING, 1987; SAWREY, 1990; ZOLLER et al., 1995) los autores entendieron que correlacionar los dibujos del nivel atómico-molecular con la ecuación química balanceada correspondiente sería la indicación de que el estudiante comprendió conceptualmente el tema de estequiometría. A raíz de los resultados que evidenciaron bajo aprendizaje conceptual se ha pregonado la necesidad de enseñar química reforzando la ejercitación sobre el nivel sub-microscópico (BUNCE; GABEL, 2002; SANGER; BADGER, 2001; SANGER et al., 2007; TABER, 2009; TALANQUER, 2011, 2012).

Siguiendo estas recomendaciones, Michael Sanger aplicó el problema conceptual de la Figura 1 para enseñar estequiometría a estudiantes de química general de la Universidad Estatal de Middle Tennessee (EEUU). En la publicación de esa experiencia (SANGER, 2005, p. 133) comentó que

[...] la mayoría de los estudiantes reconocían que la opción C es la mejor para describir la reacción química que aparece en la figura; pero, a lo largo de los años, algunos estudiantes han tratado de convencerme de que la opción $\mathrm{D}$ es también una forma correcta de describir la reacción. Mi respuesta a ellos es que a pesar de que la opción $\mathrm{D}$ es una correcta descripción del "sistema" que aparece en la figura, la opción C es la mejor descripción para la reacción química ya que no incluye especies no reactivas en la ecuación química. Yo también les señalo que la opción $\mathrm{D}$ sugiere que la proporción de combinación X:Y es 3:8 en lugar de 1:2.

Esta descripción de la interpretación diferente que hacen el experto docente o sus estudiantes novatos no fue analizada en el artículo del propio Sanger (2005), pero resulta muy oportuna para ser interpretada desde nuestro enfoque, como se verá más adelante.

Con el advenimiento de la computación muchos docentes e investigadores supusieron que mostrar movimientos con dibujos del nivel particulado de la materia mejoraría la comprensión de los estudiantes acerca de los procesos químicos en el nivel sub-microscópico (TALANQUER, 2011). Sin embargo, no se han reportado demasiados éxitos hasta el momento (GILBERT; TREAGUST, 2009; SANGER et al., 2007; VAN SOMEREN et al., 1998). La dicotomía entre aprendizaje conceptual y aprendizaje algorítmico continúa siendo una tensión propia de la enseñanza de la química (TALANQUER, 2012). 


\section{Errores por transferencia entre formatos sintácticos}

La pregunta que surge en base a lo expuesto anteriormente es si se trata de errores verdaderamente "conceptuales" o de otro tipo de errores. Nuestras investigaciones sobre obstáculos en el aprendizaje de química han revelado posibles orígenes de errores de estudiantes debidos a la complejidad de los lenguajes químicos expertos que les presentamos (GALAGOVSKY, 2008; GALAGOVSKY; BEKERMAN, 2009; GALAGOVSKY; BEKERMAN; DI GIACOMO, 2014; GALAGOVSKY; DI GIACOMO; CASTELO, 2009; GALAGOVSKY et al., 2003). Desde este enfoque teórico proponemos que esos resultados categorizados como "errores conceptuales" de los estudiantes en el tema estequiometría resultarían de transferencias erróneas de formatos sintácticos especificos entre lenguajes químicos expertos.

Para analizar las respuestas de los estudiantes desde este nuevo enfoque, se requiere reconsiderar el mencionado Triángulo de Johnstone (JOHNSTONE, 1991, 1993, 2000) sobre los niveles macro, micro y simbólico de la química; es necesario desarmarlo e interpelarlo ontológicamente. Coincidimos con Labarca (2009) en que la química como ciencia posee dos niveles ontológicos: el nivel macroscópico y el microscópico. El primero es el nivel de los fenómenos perceptibles; mientras que el segundo es el nivel de los modelos científicos que permiten interpretar a nivel atómico-molecular dichos fenómenos. Es decir, el mundo microscópico está mediado por la tecnología, por el conocimiento y por los modelos vigentes en cada época histórica de la humanidad. La química, como disciplina científica a ser comunicada requiere, además, de un plano lingüístico que queda constituido en el "discurso químico". Es en este plano en el que se proponen elementos retóricos de nivel simbólico para expresar los modelos, las interpretaciones y las explicaciones del nivel sub-microscópico, así como la descripción de los fenómenos del nivel macroscópico. Esta reconsideración del Triángulo de Johnstone se presenta en la Figura 3.

Figura 3. Análisis ontológico-lingüístico de los tres niveles de la química (macro, micro y simbólico), que modifican el conocido Triángulo de Johnstone

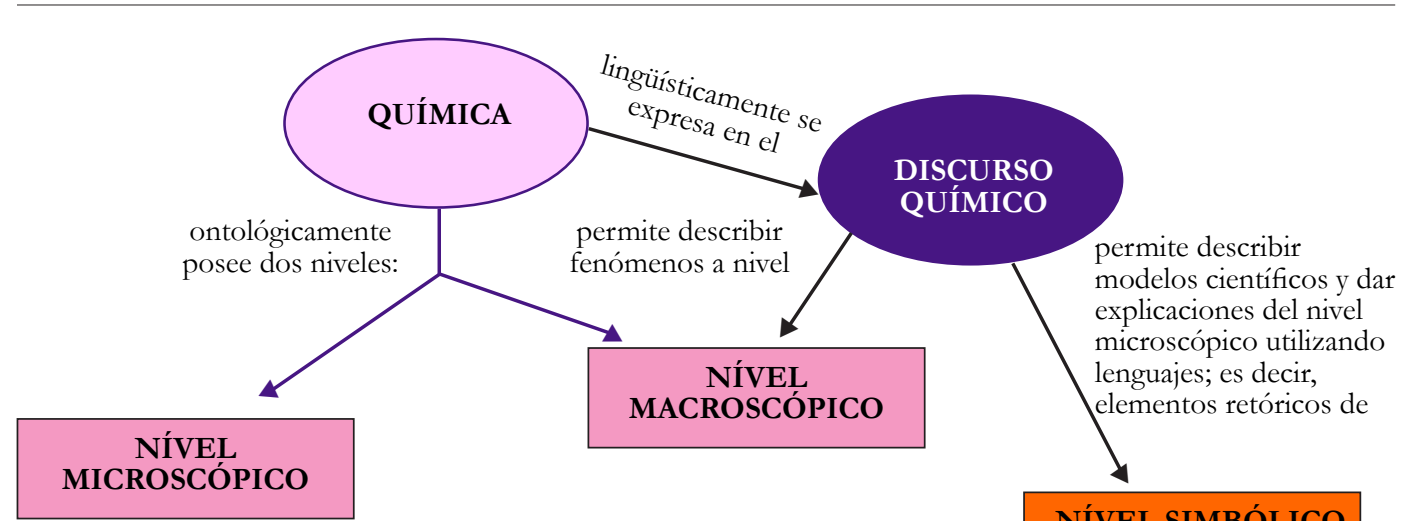

NÍVEL SIMBÓLICO

Fuente: Elaborada por los autores. 
Dado el carácter simbólico de todos los lenguajes, sostenemos que los dibujos que involucran el nivel atómico-molecular que Johnstone ha categorizado como "nivel sub-microscópico de la química" deberían ser considerados un subconjunto particular del lenguaje gráfico (GALAGOVSKY et al., 2003). Esta afirmación que encuadra a todos los lenguajes químicos en el nivel simbólico reubica a los dibujos que describen el comportamiento de partículas como un caso particular de lenguaje gráfico; se trataría de ideas simplificadas que representan gráficamente partes de modelos científicos.

Está claro que para entender dichos dibujos es necesario comprender sus códigos y formatos sintácticos específicos (símbolos), consensuados por expertos. Comprender química requiere dar un significado apropiado a cada uno de los lenguajes en que se expresa su discurso científico (GALAGOVSKY et al., 2014). Por lo tanto, en este caso, comprender estequiometría requiere diferenciar lenguajes expertos con las particularidades sintácticas especiales que diferencian a sus conceptos principales: reacción química, ecuación química balanceada y fórmulas químicas (Figura 4).

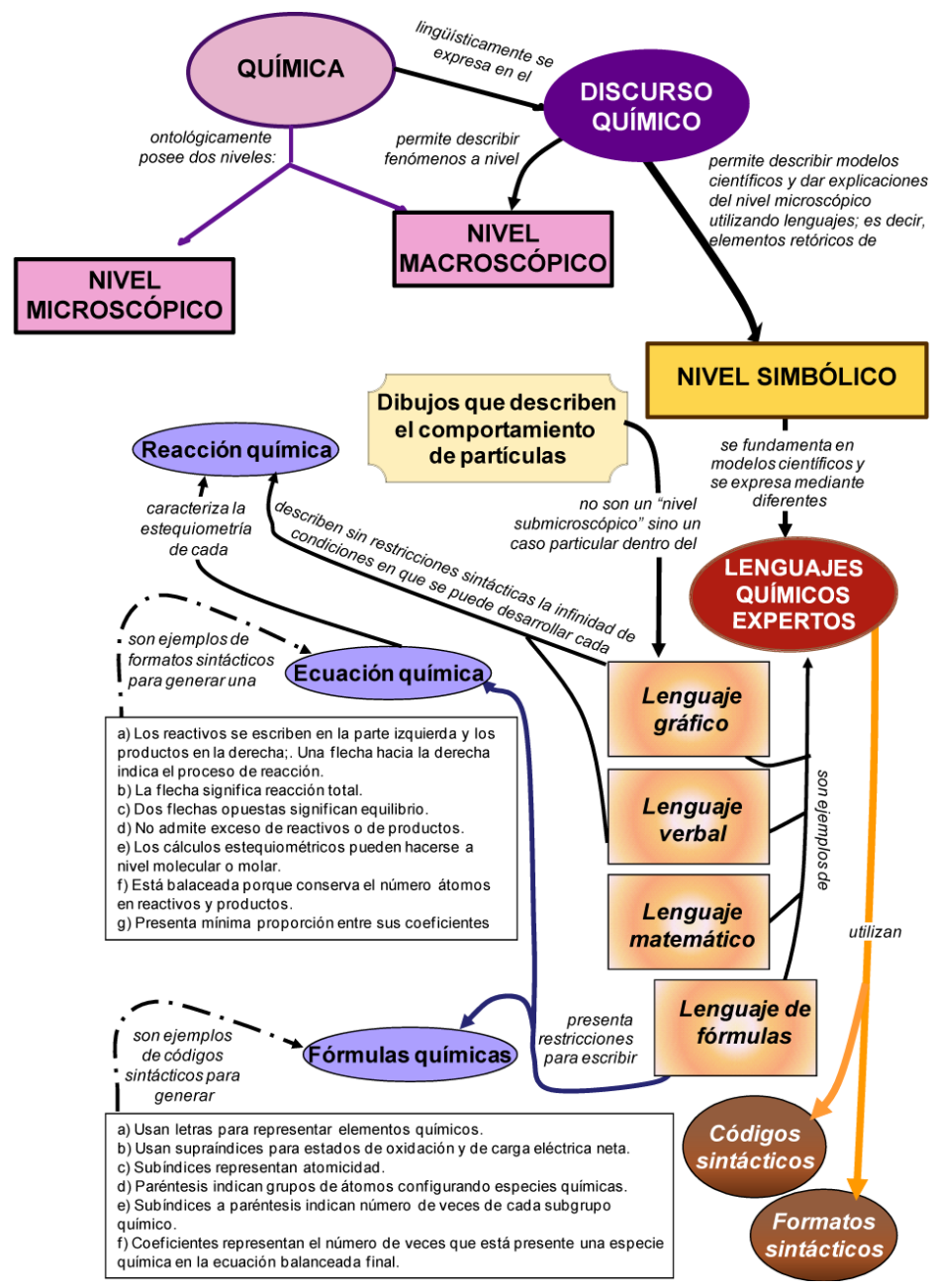

Figura 4. Marco teórico propuesto. Los errores que los estudiantes cometen sobre estequiometría serían derivados de transferencias entre lenguajes químicos expertos que tienen diferentes códigos y formatos sintácticos específicos.

Fuente: Elaborada por los autores. 
A continuación se analizarán por un lado los diversos lenguajes con los cuales se comunica el tema de estequiometría y sus conceptos claves. En segundo lugar, se evaluará cómo estos lenguajes se relacionarían con los posibles errores subyacentes a las respuestas de los estudiantes que hubieran elegido las diversas opciones planteadas en los problemas conceptuales de las Figuras 1 y 2. Descubriremos cómo la aplicación o traducción de códigos y formatos sintácticos específicos de un lenguaje químico a otro pudieron haber sido el origen de los errores de los estudiantes.

\section{Códigos y formatos sintácticos específicos de cada lenguaje químico}

Para analizar las respuestas de los estudiantes deberemos primero diferenciar los códigos y formatos sintácticos presentes en los enunciados de los problemas planteados como tradicional o conceptual en las Figuras 1 y 2.

Por un lado, se presentan los enunciados de los problemas "tradicionales" en lenguaje verbal. Este lenguaje permite describir diversas reacciones iniciales y finales (situaciones en reactores), relatando la presencia de reactivos y la existencia de reactivos en exceso. Incluso, aunque este no es el caso de las Figuras 1 y 2, los enunciados de problemas de estequiometría en lenguaje verbal permiten hacer referencia a condiciones de reacción, impurezas, porcentajes de conversión de la reacción, condiciones de equilibrio, etc.

Por otro lado, para representar mediante dibujos esquemáticos lo que ocurriría si se pudieran "ver átomos y moléculas" dentro del reactor, el único requerimiento será utilizar códigos gráficos para representar distintos tipos de átomos y guardar la proporción de las diferentes especies químicas presentes en el reactor al inicio y al final de la reacción, respetando exceso de reactivos, impurezas y conversión total o parcial de la reacción.

Es decir, tanto el lenguaje verbal como el gráfico permiten descripciones reales de reacciones químicas, lo cual es muy diferente a la situación del lenguaje de fórmulas químicas. En el lenguaje de fórmulas sólo se admite la descripción de una reacción mediante su ecuación química balanceada. De esta forma sólo deben expresarse los reactivos y productos puros, en una proporción tal que se conserve el número de átomos de cada tipo a la derecha y a la izquierda de la flecha que representa al proceso de reacción química de conversión total.

Las descripciones en lenguajes verbal o gráfico remiten a un antes y un después de una reacción química en un único reactor con sus infinitas posibilidades de proporción de reactivos, condiciones de reacción, estado de pureza de reactivos, etc. Sin embargo, en la descripción en lenguaje gráfico mediante esquemas de partículas las representaciones del "antes y el después" de la reacción están dibujadas separadas por una flecha, como si se tratara de los "reactivos y productos" de una ecuación química del lenguaje de fórmulas.

Este estilo ambiguo de presentar dibujos simbólicos del nivel particulado de la materia para estequiometría solapando formatos sintácticos propios del lenguaje verbal con aquellos del lenguaje de fórmulas puede generar confusión para los estudiantes. Por un lado, podrían producirse errores por transferencia de códigos o formatos sintácticos entre lenguajes, como se discutirá a continuación. Por otro lado, podrían contribuir a que los estudiantes no discriminen entre los conceptos de conservación de la masa aplicados a la estequiometría. Esto se acentúa aún más ya que la ley de conservación de la masa remite a sistemas macroscópicos por su origen 
histórico (GALAGOVSKY; DI GIACOMO; ALÍ, 2013). Contrariamente a los lenguajes verbal y gráfico, el lenguaje de fórmulas químicas tiene fuertes restricciones sintácticas que se detallan en los Cuadros 1 y 2, y se han incluido en la red de la Figura 4. Estas precisiones de códigos y formatos sintácticos serán utilizadas en el próximo apartado para mostrar cómo su aplicación inapropiada a otros lenguajes constituiría el fundamento de los errores de los estudiantes en los problemas "conceptuales" de las Figuras 1 y 2.

Cuadro 1. Códigos sintácticos para el lenguaje de formulas químicas

a. Con letras se representa cada elemento químico; puede o no indicarse estado de oxidación.

b. Los supraíndices con números y signos (más o menos), muestran estados de oxidación, carga eléctrica neta para un átomo o conjunto de átomos que forman iones.

c. Los subíndices representan el número de átomos presentes de cada elemento químico.

d. Paréntesis indican que el arreglo de átomos encerrados en él forman una especie química.

e. Subíndices a la derecha abajo de un paréntesis indican el número de veces que un subgrupo químico está presente en la especie química total.

f. Los coeficientes representan el número de veces que está presente una especie química en la ecuación balanceada final. Los coeficientes deben ser números enteros.

Fuente: Elaborado por los autores.

Cuadro 2. Formatos sintácticos (FS) para ecuaciones químicas

FS-1. Un cambio químico implica reordenamientos en la combinación de elementos químicos, con o sin transferencia de electrones entre especies químicas reactivas; se identifica como reacción química.

FS-2. Una reacción química se escribe mediante una ecuación química balanceada.

FS-3. Los reactivos se escriben en la parte izquierda de la ecuación química balanceada y los productos en la parte derecha; una flecha hacia la derecha indica el proceso de reacción entre reactivos y productos.

FS-4. Para los cálculos estequiométricos la flecha significa reacción irreversible y total (reactivos se convierten en productos en un $100 \%$ ).

FS-5. Dos flechas opuestas significan que la reacción llega a un equilibrio.

FS-6. La ecuación química balanceada no admite exceso de reactivos o de productos.

FS-7. Los cálculos estequiométricos pueden presentarse a nivel molecular o molar.

FS-8. En la ecuación química balanceada se conserva la masa: el número de veces en que se encuentra cada elemento químico como reactivo o producto, independientemente de su estado de agregación.

FS-9. Una ecuación química balanceada debe mostrar la mínima proporción de coeficientes que permitan conservar el número total de cada tipo de átomos involucrados en la reacción química.

FS-10. La masa total se conserva en recipientes cerrados donde ocurren reacciones químicas (ley de conservación de la masa, macroscópica).

Fuente: Elaborado por los autores. 


\section{Errores por transferencias inapropiadas entre lenguajes}

Se ha planteado que el discurso químico presenta laxitud sintáctica para describir problemas de estequiometría en lenguajes verbal o gráfico, pero exigencias sintácticas precisas en el lenguaje de fórmulas químicas. Cabe interpretar, entonces, que los estudiantes novatos no comprendiendo cabalmente esta discriminación entre lenguajes, puedan "razonar lógicamente" para finalmente elegir incorrectamente algunas de las opciones de los problemas "conceptuales" de las Figuras 1 y 2.

En los Cuadros 3 y 4 mostramos algunos de estos posibles razonamientos, haciendo mención a los formatos sintácticos presentes en las ecuaciones químicas (FS-1 a FS-10, Cuadro 2) que se habrían tenido en cuenta para seleccionar cada opción.

Cuadro 3. Posibles respuestas de los estudiantes novatos derivadas de transferencias equivocadas entre códigos y formatos sintácticos de diferentes lenguajes, para el problema "conceptual" de la Figura 1.

Respuesta A: En una ecuación bien escrita no deben sobrar reactivos (FS-6). Hay que contar para ver que lo que está escrito al principio se conserve al final (FS-10). Si sobran círculos se debe a que todavía no terminaron de reaccionar, porque al final todo tiene que reaccionar (FS-4).

Respuesta B: Hay tres cuadrados, al final se juntan con seis redondos pero las partículas que "sobran" no se tienen que contar (FS-6). Al final todo tiene que reaccionar (FS-4).

Respuesta D: Hay que poner en las fórmulas químicas todo lo que se presenta en el dibujo.

Fuente: Elaborado por los autores.

Obsérvese en el Cuadro 3 que para elegir la opción correcta (respuesta C de la Figura 1) debería realizarse una estricta y compleja secuencia de pasos electivos:

a) Fijarse en el dibujo cuál es la fórmula del producto nuevo. Esto implica reconocer que puede haber reactivos que no han reaccionado, detectándose así el reactivo limitante. Si no se observa que hay reactivos en exceso, igualmente es necesario chequear que haya el mismo número de tipos de elementos dibujados en el estado inicial.

b) Luego, para escribir la ecuación química balanceada hay que ver la atomicidad en el dibujo del producto y buscar coeficientes que multiplicados por el subíndice de cada átomo den un resultado balanceado (misma cantidad de átomos en reactivos y productos).

c) Luego se construye la ecuación química, con la mínima proporción de coeficientes, sin importar que en el dibujo haya elementos gráficos sobrantes.

Nótese también que la opción D fue siempre la más elegida como respuesta al problema "conceptual" de la Figura 1. La misma corresponde a una transferencia literal del lenguaje verbal o gráfico, al lenguaje de fórmulas. Es decir, para el lenguaje de fórmulas la ecuación química requiere un balance de la cantidad de cada tipo de átomos a cada lado de la flecha. Esto implica una reflexión epistemológica para diferenciar conceptual y experimentalmente la idea de conservación de la masa en sus versiones macroscópica o atómico-molecular (sub-microscópica). Esta necesaria diferenciación es la cuestión subyacente a la discusión relatada más arriba entre Michael Sanger (2005) y sus estudiantes. 
Cuadro 4. Posibles respuestas para el problema "conceptual” (Figura 2) derivadas de transferencias equivocadas entre códigos y formatos sintácticos de diferentes lenguajes

Respuesta a: Se combinan todas las especies para que no sobre ninguna (FS-10). Así si son seis blancas y doce negras, la fórmula química del producto debe ser una blanca con dos negras, porque todo debe reaccionar (FS-4).

Respuesta b: En el estado final, los átomos se conservan, sólo se "reordenan"; cambian sus posiciones unos con respecto a otros (FS-1). El problema dice que es una mezcla final, y los dibujos "a", "b" y "d" no son una mezcla.

Respuesta c: En la ecuación química balanceada se muestra que reacciona todo y no sobra nada (FS-4). Hay seis blancos inicialmente, se completa con los negros necesarios, porque el dibujo del estado inicial muestra sólo una parte de las partículas que hay.

Respuesta d: La ecuación química dice que se forman dos partículas de $\mathrm{SO}_{3}$.

Fuente: Elaborado por los autores.

La elección de la respuesta c en el Cuadro 4 implica que el suponer que habría muchas más partículas no dibujadas se correspondería con una transferencia del lenguaje verbal. Esto se debe a que si en la pregunta "tradicional” (Figura 2) decía que había exceso de azufre (S), entonces aquí podría haber exceso de oxígeno $(\mathrm{O})$. Asimismo, la elección de la respuesta (d) involucra una transferencia del lenguaje de fórmulas al lenguaje gráfico.

Obsérvese que para elegir la opción correcta (c) para el problema "conceptual" de la Figura 2 debería realizarse una estricta secuencia de pasos electivos:

a) Fijarse en la ecuación química balanceada cuál es la fórmula del producto final y encontrar ese producto en el esquema de partículas, quedando posibles las opciones "c", "d" y "e".

b) Contar cuántos átomos de cada tipo hay dibujados en el esquema final y cuántos en el sistema inicial, y compararlos.

c) Descartar si sobran átomos de ambos reactivos en el sistema inicial respecto del sistema final, porque no habría conservación de la masa (opción “d”).

d) Descartar si faltan átomos de algún reactivo en el sistema inicial respecto de los existentes en el sistema final, porque no habría conservación de la masa (opción “c”).

e) Aceptar aquel dibujo que conserve la masa entre sistema inicial y final, aunque pueda haber reactivos sobrantes (que no han reaccionado). Esto implica que el otro reactivo es el limitante.

\section{Conclusiones}

El análisis realizado da cuenta de la complejidad de los lenguajes químicos para el tema de estequiometría. Los expertos los utilizamos complementariamente, pero cada uno de ellos expresa sólo parte de las interpretaciones simbólicas con las que la química modela los fenómenos macroscópicos. Así, los docentes planteamos a los estudiantes problemas muy variados sin percatarnos que aún los discursos que nos parecen sencillos pueden estar originándoles grandes desafíos que se convierten en obstáculos de sus aprendizajes. 
Nuestra propuesta resumida en la red conceptual de la Figura 4 permite hacer visibles los códigos y formatos sintácticos de los diferentes lenguajes químicos involucrados en el tema de estequiometría. El punto central del análisis marca tres puntos importantes: por un lado, que una reacción química, en tanto fenómeno macroscópico, puede ser descrita en lenguaje verbal sin restricciones sintácticas, por cuanto habría infinidad de situaciones reales en la que se intente realizar una dada reacción química. Por otro lado, que la estequiometría de dicha reacción química sólo se describe en lenguaje de fórmulas químicas, y tiene severas restricciones sobre cómo debe expresarse. Finalmente, no se ha consensuado en la comunidad de expertos si el lenguaje gráfico que representaría una dada reacción química mediante la visualización de "partículas" de nivel atómico-molecular debería o no respetar las restricciones del lenguaje de fórmulas (simbolizando estrictamente las proporciones de la ecuación química balanceada), o la libertad sintáctica de la descripción verbal de una situación macroscópica en la que ciertas cantidades variables de reactivos se someten a unas dadas condiciones - variadas - de reacción.

Así, esta tolerada indeterminación para los códigos y formatos sintácticos de los dibujos que representan las "partículas" intervinientes en una reacción química daría lugar a fallas en la comunicación entre los expertos-docentes y los estudiantes-novatos. Un ejemplo de esta ambigüedad comunicativa se ha presentado en la transcripción del comentario de Michael Sanger $(2005)^{2}$.

$\mathrm{El}$ análisis propuesto es también aplicable para interpretar el origen de las desazones de algunos docentes investigadores frente a las equivocaciones de sus estudiantes (LEE, 1999; MULFORD; ROBINSON, 2002; NICOLL, 2003; WILLIAMSON; HUFFMAN; PECK, 2004).

\section{Reflexiones finales}

Los investigadores que propusieron diferenciar entre problemas de tipo "algorítmico" y problemas de tipo "conceptual” como los presentados en las Figuras 1 y 2 concluyeron que los estudiantes resuelven mejor el primer tipo de problemas pues están mejor entrenados en ellos.

Nuestra propuesta considera a los lenguajes expertos como mediadores de la comunicación entre expertos y novatos y lleva a reflexionar sobre la complejidad del discurso científico que usamos los docentes de química, tanto para enseñar como para evaluar.

En este trabajo hemos mostrado cómo no resulta una tarea evidente comprender que hay códigos y formatos sintácticos específicos para cada lenguaje químico para el tema específico de estequiometría. La invisibilidad de este problema epistemológico para los docentes resulta en la construcción de discursos que encriptan obstáculos de aprendizaje para los estudiantes. Desde esta perspectiva, los errores conceptuales evidenciados en las evaluaciones que preparamos los docentes, serían indicadores de las dificultades de los estudiantes para procesar el discurso químico.

Debemos reflexionar sobre qué decimos y qué queremos significar cuando, como docentes, "hablamos química" y cuando evaluamos a nuestros estudiantes.

\footnotetext{
${ }^{2}$ Ver apartado previo "Los estudiantes no aprenden conceptualmente".
} 


\section{Referencias}

ALÍ, S. et al. Libros de texto de química: ¿fuentes de comunicación o incomunicación? Industria y Química, Buenos Aires, n. 362, p. 61-64, 2010.

BEKERMAN, D. G. La utilización de la imágen en los procesos de enseñanza y aprendizaje de química orgánica. 2006. 249 h. (Tesis doctoral) - Facultad de Farmacia y Bioquímica, Universidad de Buenos Aires, Buenos Aires, 2006.

BUNCE, D. M.; GABEL, D. J. Differential effects on the achievement of males and females of teaching the particulate nature of chemistry. Journal of Research in Science Teaching, Hoboken, v. 39, n. 10, p. 911-927, 2002.

GALAGOVSKY, L. Enseñanza de las ciencias naturales: un desafío a nivel mundial. El caso particular de enseñanza de la química. Proyecciones, Buenos Aires, v. 6, n. 2, p. 23-36, 2008.

GALAGOVSKY, L.; BEKERMAN, D. La química y sus lenguajes: un aporte para interpretar errores de los estudiantes. Revista Electrónica de Enseñanza de las Ciencias, Vigo, v. 8, n. 3, p. 952-975, 2009.

GALAGOVSKY, L.; BEKERMAN, D.; DI GIACOMO, M. A. Enseñanza de la química: lenguajes expertos como obstáculos de aprendizaje. In: MERINO, C.; ARELLANO, M.; ADÚRIZ-BRAVO, A. (Ed.). Avances en didáctica de la química: modelos y lenguajes. Valparaíso: Pontificia Universidad Católica de Valparaíso, 2014. p. 107-118.

GALAGOVSKY, L.; DI GIACOMO, M.; ALÍ, S. Estequiometría y ley de conservación de la masa: lo que puede oculta la simplificación del discurso experto. (En prensa).

GALAGOVSKY, L.; DI GIACOMO, M. A.; CASTELO, V. Modelos vs. dibujos: el caso de la enseñanza de fuerzas intermoleculares. Revista Electrónica de Enseñanza de las Ciencias, Vigo, v. 8, n. 1, p. 1-22, 2009.

GALAGOVSKY, L. BEKERMAN, D.; DI GIACOMO, M. A; ALÍ, S. Algunas reflexiones sobre la distancia entre "hablar química" y "comprender química". Ciência \& Educação, Bauru, v. 20, n. 4, 2014.

GALAGOVSKY, L. et al. Representaciones mentales, lenguajes y códigos en la enseñanza de ciencias naturales: un ejemplo para el aprendizaje del concepto de reacción química a partir del concepto de mezcla. Enseñanza de las Ciencias, Barcelona, v. 21, n. 1, p. 107-121, 2003.

GILBERT, J. K.; TREAGUST, D. (Ed.). Multiple representations in chemical education. Dordrecht: Springer, 2009.

JOHNSTONE, A. H. The development of chemistry teaching: a changing response to a changing demand. Journal of Chemical Education, Washington, v. 70, n. 9, p. 701-705, 1993.

. Teaching of chemistry: logical or psychological? Chemistry Education: research and practice, Cambridge, v. 1, n. 1, p. 9-15, 2000. 
Why is science difficult to learn?: things are seldom what they seem. Journal of Computer Assisted Learning, Chichester, v. 7, n. 2, 75-83, 1991.

LABARCA, M. Acerca de la naturaleza de la química: algunos comentarios. Educación en la Química, Buenos Aires, v. 15, n. 2, p. 1-12, 2009.

LEE, K-W. L. A comparison of university lecturers' and pre-service teachers' understanding of a chemical reaction at the particulate level. Journal of Chemical Education, Washington, v. 76, n. 7, p. 1008-1012, 1999.

LEMKE, J. L. Aprender a hablar ciencia: lenguaje, aprendizaje y valores. Barcelona: Paidós Ibérica, 1997.

MULFORD, D. R.; ROBINSON, W. R. An inventory for alternative conceptions among first semester General Chemistry students. Journal of Chemical Education, Washington, v. 79, n. 6, p. 739-744, 2002.

NAKHLEH. M. B.; MITCHELL, R. C. Concept learning versus problem solving: there is a difference. Journal of Chemical Education, Washington, v. 70, n. 3, p. 190-192, 1993.

NICOLL, G. A qualitative investigation of undergraduate chemistry students' macroscopic interpretation of the submicroscopic structure of molecules. Journal of Chemical

Education, Washington, v. 80, n. 2, p. 205-213, 2003.

NURRENBERN, S.; PICKERING, M. Concept learning versus problem solving: is there a difference? Journal of Chemical Education, Washington, v. 64, n. 6, p. 508-510, 1987.

SANGER, M. J. Evaluating students'conceptual understanding of balanced equations and stoichiometric ratios using a particulate drawing. Journal of Chemical Education, Washington, v. 82, n. 1, p. 131-134, 2005.

SANGER, M.; BADGER, S. Use of simultaneous- synchronized macroscopic, microscopic, and symbolic representations to enhance the teaching and learning of chemical concepts. Journal of Chemical Education, Washington, v. 74, n. 3, p. 330-334, 2001.

SANGER, M. J. et al. Concept learning vs. problem solving: does particle motion have and effect? Journal of Chemical Education, Washington, v. 84, n. 5, p. 875-879, 2007.

SAWREY, B. A. Concept learning versus problem solving: revisited. Journal of Chemical Education, Washington, v. 67, n. 3, p. 253-254, 1990.

SHANNON, C. E.; WEAVER, W. The mathematical theory of communication. Urbana: University of Illinois Press, 1949.

TABER, K. S. Learning at the symbolic level. In: GILBERT, J. K.; TREAGUST, D. (Ed.). Multiple representations in chemical education. Dordrecht: Springer, 2009. p. 75-108.

TALANQUER, V. Chemistry education: ten dichotomies we live by. Journal of Chemical Education, Washington, v. 89, n. 11, p. 1340-1344, 2012. Macro, submicro, and symbolic?: the many faces of the chemistry triplet.

International Journal of Science Education, Abingdon, v. 33, n. 2, p. 179-195, 2011. 
VAN SOMEREN, M. W. et al. (Ed.). Learning with multiple representations. New York: Elsevier, 1998.

WILLIAMSON, V.; HUFFMAN, J.; PECK, L. Testing students' use of the particulate theory. Journal of Chemical Education, Washington, v. 81, n. 6, p. 891-896, 2004.

ZOLLER, U. et al. Success on algorithmic and LOCS vs. conceptual chemistry exam questions. Journal of Chemical Education, Washington, v. 72, n. 11, p. 987-989, 1995. 